Abstract 435 Table 1 Evolution of disease activity patterns from 1 to 3 years.

\begin{tabular}{|c|c|c|c|c|c|}
\hline \multicolumn{2}{|c|}{ Disease Pattern at l year, N(\%) } & \multicolumn{4}{|c|}{ Disease Pattern at 3 years, N(\%) } \\
\cline { 3 - 6 } & CQD & MDA & RRD & CAD \\
\hline $\begin{array}{c}\text { Clinical Quiescent } \\
\text { Disease (CQD) }\end{array}$ & $37(31.4)$ & $26(70.3)$ & $0(0.0)$ & $7(18.9)$ & $4(10.8)$ \\
\hline $\begin{array}{c}\text { Minimal Disease } \\
\text { Activity (MDA) }\end{array}$ & $3(2.5)$ & $2(66.7)$ & $1(33.3)$ & $0(0.0)$ & $0(0.0)$ \\
\hline $\begin{array}{c}\text { Relapsing-Remitting } \\
\text { Disease (RRD) }\end{array}$ & $30(25.4)$ & $14(46.7)$ & $0(0.0)$ & $13(43.3)$ & $3(10.0)$ \\
\hline $\begin{array}{c}\text { Chronic Active Disease } \\
\text { (CAD) }\end{array}$ & $48(40.7)$ & $17(35.4)$ & $1(2.1)$ & $14(29.2)$ & $16(33.3)$ \\
\hline TOTAL & $118(100.0)$ & $59(50.0)$ & $2(1.7)$ & $34(28.8)$ & $23(19.5)$ \\
\hline
\end{tabular}

flares in 87 patients, including 73 (56\%) renal flares. Eleven patients died during the 3 year observation period.

Conclusions This study reflects improvement in disease patterns among patients participating in an observational cohort study. Damage is largely driven by high cumulative steroid use.

\section{PROGNOSIS FOR HOSPITALISED PATIENTS WITH SYSTEMIC LUPUS ERYTHEMATOSUS IN CHINA: 5-YEAR UPDATE OF THE JIANGSU COHORT}

${ }^{1} X$ Feng*, ${ }^{2} \mathrm{P}$ Wenyou, ${ }^{3} \mathrm{~L}$ Lin, ${ }^{4} \mathrm{~W}$ Min, ${ }^{1} \mathrm{~S}$ Lingyun. 'Affiliated Drum Tower Hospital of Nanjing University Medical School, Department of Rheumatology, Nanjing, China; ${ }^{2}$ Huaian First People's Hospital, Department of Rheumatology, Huaian, China; ${ }^{3}$ Xuzhou Central Hospital, Department of Rheumatology, Xuzhou, China; ${ }^{4}$ Third Affiliated Hospital of Soochow University, Department of Rheumatology, Changzhou, China

\subsection{6/lupus-2017-000215.436}

Background and aims To identify early signs associated with poor prognosis in Chinese patients with systemic lupus erythematosus (SLE) through a large population-based follow-up study.

Methods Medical records of $>2500$ SLE patients that first hospitalised between 1999 -2009 were collected from 26 centres across Jiangsu province, China, and entered into a database. These patients were followed-up for 5 to 15 years, and those remained contact and had known survival status in 2015 were assessed for the association of factors presented at the initial hospitalisation with mortality. The independency of mortality factors was evaluated using multivariate Cox regression analysis.

Results Among 1372 patients we assessed, 92.3\% were women and $17.2 \%$ were deceased in 2015 . The main causes of death were infection, neuropsychiatric impairment, renal failure and cardiopulmonary involvement. Hazard ratios (HR) of independent predictors for mortality $(\leq 1$ year and $>1$ year, respectively) included hospital presentation of neuropsychiatric involvement (2.03 and 1.91), cardiopulmonary involvement (1.94 and 1.61) and increased serum creatinine (2.52 and 2.58). The presence of anti-dsDNA and anti-Sm antibodies indicated diverse prognosis after 1 year (HR 1.60 and 0.45 ). Treatment with cyclophosphamide was beneficial for patient's first-year outcome (HR 0.50), and anti-malarial drugs significantly reduced the risk of mortality over different time points (HR 0.48 and 0.54). SLEDAI score, proteinuria or hypocomplementemia was not independently associated with the outcome.

Conclusions SLE patients presented with vital organ damages rather than active disease at initial hospitalisation are likely to have a poor outcome. Early and effective intervention with the use of anti-malarial drugs may decrease mortality. 


\section{CONSTRUCT VALIDITY ASSESSMENT OF THE LUPUS LOW DISEASE ACTIVITY STATE (LLDAS) - A CASE BASED VALIDITY STUDY}

${ }^{1} V$ Golder ${ }^{*},{ }^{2} \mathrm{M}$ Huq, ${ }^{1} \mathrm{~K}$ Franklyn, ${ }^{2} \mathrm{~A}$ Calderone, ${ }^{3} \mathrm{~A}$ Lateef, ${ }^{4} \mathrm{CS}$ Lau, ${ }^{4} \mathrm{~A}$ Lee, ${ }^{5} \mathrm{~S}$ Navarra, ${ }^{2} \mathrm{~T}$ Godfrey, ${ }^{2} \mathrm{~S}$ Oon, ${ }^{1} \mathrm{~A}$ Hoi, ${ }^{1} \mathrm{E}$ Morand, ${ }^{2} \mathrm{M}$ Nikpour. ${ }^{1}$ School of Clinical Sciences at Monash Health, Rheumatology, Clayton, Australia; ${ }^{2}$ Melbourne University, Rheumatology, Fitzroy, Australia; ${ }^{3}$ National University Hospital, Rheumatology, Singapore, Singapore; ${ }^{4}$ Hong Kong University, Rheumatology, Hong Kong, Hong Kong S.A.R; ${ }^{5}$ University of Santo Tomas Hospital, Rheumatology, Manila, Philippines

10.1136/lupus-2017-000215.437

Background and aims To evaluate the construct validity of the Lupus Low Disease Activity State (LLDAS), a treatment target in systemic lupus erythematosus (SLE).

Methods Fifty SLE case summaries based on real patients were prepared and assessed independently for meeting the operational definition of LLDAS. Fifty international rheumatologists with expertise in SLE, but with no prior involvement in the LLDAS project, responded to a survey in which they were asked to categorise the disease activity state of each case as remission, low, moderate or high. Agreement between expert opinion and LLDAS was assessed using Cohen's Kappa.

Results Overall agreement between expert opinion and the operational definition of LLDAS was $77.96 \% \quad(95 \%$ CI $76.34 \%-79.58 \%)$, with a Cohen's Kappa of 0.57 (95\% CI $0.55-0.61$ ). Of the cases (22 of 50 ) that fulfilled the operational definition of LLDAS, only 5.34\% (59 of $22 \times 50)$ of responses classified the cases as moderate/high activity. Of the cases that did not fulfil the operational definition of LLDAS (28 of 50$), 35.14 \%$ (492 of $28 \times 50)$ of responses classified the cases as remission/low activity. Common reasons for discordance were assignment to remission/low activity of cases with higher corticosteroid doses than defined in LLDAS (prednisolone $\leq 7.5 \mathrm{mg}$ ) or with SLEDAI-2K $>4$ due to serological activity (high anti-dsDNA antibody and/or low complement).

Conclusions LLDAS has good construct validity with high overall agreement between the operational definition of LLDAS and expert opinion. Discordance of results suggests that the operational definition of LLDAS is more stringent than expert opinion at defining a low disease activity state.

\section{COMPARISON QUALITY OF LIFE OF PATIENT WITH SYSTEMIC LUPUS ERYTHEMATOSUS AND SYSTEMIC SCLEROSIS}

${ }^{1}$ L Hamijoyo*, ${ }^{2}$ II Hidayat, ${ }^{2} \mathrm{BS}$ Suryajaya, ${ }^{2} \mathrm{VN}$ Moenardi, ${ }^{1} \mathrm{~S}$ Dewi, ${ }^{1} \mathrm{R}$ G Wachjudi. ${ }^{1}$ Universitas Padjadjaran, Internal Medicine, Bandung, Indonesia; ${ }^{2}$ Universitas Padjadjaran, Immunology Study Group- Faculty of Medicine, Bandung, Indonesia

10.1136/lupus-2017-000215.438

Background and aims Patients with autoimmune patients have to deal with their chronic disease, and it affects their quality of life. This study was aimed to compare the quality of life (QOL) of patient with systemic lupus erythematosus (SLE) and systemic sclerosis (SSc).

Methods This study was cross sectional study, done in rheumatology outpatient clinic Hasan Sadikin Hospital Bandung, Indonesia in 2015-2016. It was conducted by asking patients diagnosed of SLE and SSc to fill the SF-36 form. Baseline characteristic, including age, gender, and duration of disease, were collected during the visit. The Mann-Whitney U test was used to analyse the comparison.

Results One hundred seventy patients were involved into this study, consisted of 127 SLE patients and 43 SSc patients, mostly female 162 patients (96.3\%). Median of age was 34 (19 to 62) year and 40 (16 to 69) year (min-max), and duration of disease was $72(3-252)$ and 36 (1-228) months (minmax), consecutively for SLE and SSc

All sections of quality of life in patient with SLE was significantly higher than patients with SSc with $\mathrm{p}<0.001$, with exception on mental component score (MCS) was not significantly different in SLE compared to SSc $(\mathrm{p}=0.062)$.

Conclusions The quality of life of SLE patients is significantly higher than patients with SSc, except mental component score was not significantly different.

\section{CEREBROVASCULAR EVENTS IN SYSTEMIC LUPUS ERYTHEMATOSUS: RESULTS FROM AN INTERNATIONAL, INCEPTION COHORT STUDY}

JG Hanly for SLICC*. Dalhousie University, Rheumatology, Halifax- Nova Scotia, Canada

\subsection{6/lupus-2017-000215.439}

Background and aims To determine the frequency, associations and outcomes of cerebrovascular events (CerVEs) in a multiethnic/racial, prospective, inception cohort of SLE patients.

Methods Patients were assessed annually for 19 neuropsychiatric events including 5 types of CerVEs: (i) Stroke; (ii) Transient ischemia; (iii) Chronic multifocal ischemia; (iv) Subarachnoid and intracranial haemorrhage; (v) Sinus thrombosis. Global disease activity (SLEDAI-2K), SLICC/ACR damage index (SDI) and SF-36 subscale, mental (MCS) and physical (PCS) component summary scores were collected. Time to event, linear and logistic regressions and multi-state models were used as appropriate.

Results Of 1826 SLE patients, $88.8 \%$ were female, $48.8 \%$ Caucasian, mean \pm SD age $35.1 \pm 13.3$ years, disease duration $5.6 \pm 4.2$ months and follow-up $6.6 \pm 4.1$ years. CerVEs were the fourth most frequent NP event: 82/1,826 (4.5\%) patients had 109 events and 103/109 (94.5\%) were attributed to SLE. The predominant events were stroke [60/109 (55.0\%)] and transient ischemia [28/109 (25.7\%)]. CerVEs were associated with NP events attributed to SLE (HR (95\% CI): (3.16; 1.73 $5.75)$, non-SLE $(2.60 ; 1.49-4.51)(\mathrm{p}<0.001)$, African ancestry at US SLICC sites $(2.04 ; 1.01-4.13)(p=0.047)$ and organ damage $(p=0.041)$. Lupus anticoagulant increased the risk of first CerVE (1.77; 0.99-3.16). Physician assessment indicated resolution or improvement in the majority but patients reported a sustained reduction in SF-36 summary and subscale scores following CerVEs $(\mathrm{p}<0.0001)$.

Conclusions CerVEs, the fourth most frequent NP event in SLE, are usually attributable to lupus early in the disease course. In contrast to good physician reported outcomes, patients report a sustained reduction in health related quality of life following CerVEs. 